\title{
Mitigation of load side harmonic distortion in standalone photovoltaic based microgrid
}

\author{
Adel Aljwary ${ }^{1}$, Ziyodulla Yusupov ${ }^{2,3^{*}}$, Olimjon Toirov ${ }^{4}$, and Rustam Shokirov ${ }^{4}$ \\ ${ }^{1}$ Department of Electromechanical Engineering, University of Technology, Baghdad, Iraq \\ ${ }^{2}$ Department of Electrical-Electronics Engineering, Karabuk University, Karabuk, Turkey \\ ${ }^{3}$ Power Supply and Renewable Energy Sources, Tashkent Institute of Irrigation and Agricultural \\ Mechanization Engineers, 100000 Tashkent, Uzbekistan \\ ${ }^{4}$ Department of Electric Machine Engineering, Tashkent State Technical University named after \\ Islam Karimov, Tashkent, Uzbekistan
}

\begin{abstract}
Photovoltaic (PV) system as one part of distributed energy resources is becoming an alternative for low and medium distribution network of microgrid. By the reason of a wide implementation of power electronic and non-linear loads, harmonics distortion is one of the main problems for the power systems. There are several filter types to mitigate the harmonics. The passive filter is distinguished by its simplicity and economical options from another filters. In this paper, the passive single tuned filter (STF) is used to minimize harmonics distortion in standalone PV based microgrid. A solar PV array is modelled as an ideal single diode model (ISDM) and used to supply electrical power to an AC load. The simulation results are executed on MATLAB/Simulink show that STF is effective in mitigating the voltage total harmonic distortion (VTHD) and the current total harmonic distortion (ITHD) with enhancing the output power quality.
\end{abstract}

\section{Introduction}

In last decades, for some of reasons, including the limited resources of fossil fuels, new environmental policies, global warming effects, and the intermittency of renewable energy sources, improving of electric energy production has become a top priority around the world $[1,2]$. In recent years, impetuous infiltration of renewable energy sources (RES) to electrical power system, the development of power electronic system and implementation of new information and communication technology led to the concept of Smart Grid (SG). Microgrid (MG) as a key component of SG can be functioned in small-scale version of electric power system in stand-alone mode or in grid-connected mode, i.e., with main grid [3]. The microgrid term refers to the concept of medium/low voltage distribution networks of power subsystem associated with a group of distributed energy resources (DER), energy storage devices and loads [4].

Photovoltaic (PV) system as one part of DER is becoming an alternative for low and medium distribution network of MG. The PV power output is specified by the potential

* Corresponding author: ziyadullayusupov@karabuk.edu.tr 
difference of the terminals of the panel. The maximum power point (MPP) of the PV varies with solar irradiance and temperature. For the V-I and V-P characteristic curves of PV there is only one point - the maximum power point (MPP) at which the highest power is supplied to the load. The efficiency of the PV is the highest at the MPP. There are many methods have been derived to calculate MPP [5]. A dynamic MPPT is implemented in PV for microgrids [6]. Abdelsalam et.al. are offered high performance adaptive perturb and observe (P\&O) technique in [7]. Theoretical and experimental analysis of genetic algorithm control method for maximum power point tracking (MPPT) are presented by Hadji et al. [8]. In MPPT, most control schema use the $\mathrm{P} \& \mathrm{O}$ technique because it is easy to implement. MPPT efficiency is analyzed using P\&O algorithm and a new P\&O technique for digital implementation has been proposed in [9] that can improve the steady state MPPT efficiency. By the reason of a wide implementation of power electronic and non-linear loads, harmonics distortion is one of the main problems for the power systems. The multiples frequencies of the rated frequency of the grid are the voltage and current harmonics. Harmonics combined with the rated frequency component of voltage and current can produce waveform distortion. The distortion exists because of currents of harmonics generated by non-linear loads supplied by the grid. The distortion in voltage is due to the flowing of the currents of harmonics through the grid impedance $[10,11]$. In [12] PV solar module is modelled as an ideal single diode model (ISDM) and used to supply electrical power to an AC load, the output voltage of the PV is boosted by a DC-DC boost converter which is controlled by a pulse width modulated (PWM) duty cycle. To get the maximum power output from the solar $\mathrm{PV}$ with variation of irradiance and temperature conditions a P\&O technique is applied for MPPT. The DC-DC converter output voltage is inverted to AC using a full bridge (FB) inverter controlled by a PWM controller. The output voltage and current waveforms are distorted as they are polluted with harmonics due to the nonlinearity of the power electronics converters. A series passive single tuned filter (STF) which is a simple and an economical option is used for harmonic mitigation.

\section{Methodology}

\subsection{Block diagram of standalone PV system}

The simulated PV standalone solar system is shown in Fig. 1. The P\&O algorithm of MPPT is tracking the PV maximum power operating point for all environment conditions (irradiance and temperature), the calculated duty cycle is pulse width modulated to control the DC-DC boost converter to step up the PV maximum operating point voltage to the required input operating voltage of the DC-AC FB inverter which is controlled by a PWM controller. The voltage and current output of the inverter is distorted with harmonics and injected to the load.

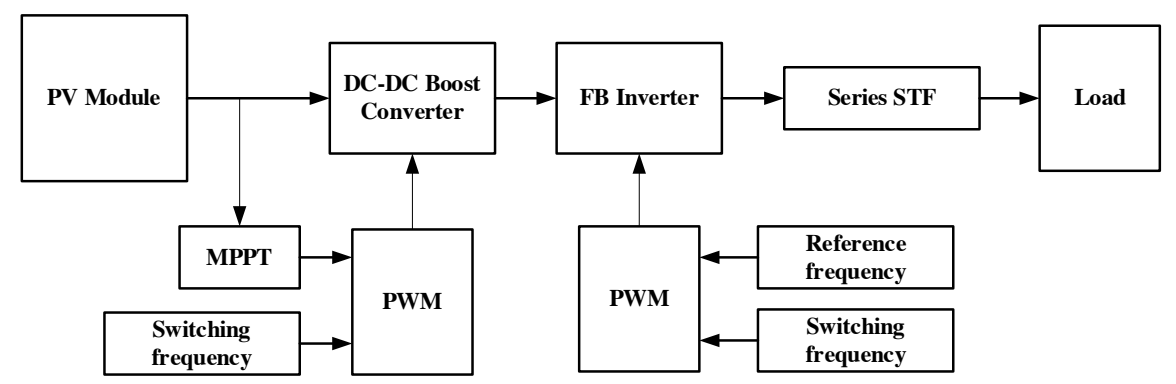

Fig. 1. Block diagram of simulated system 


\subsection{Passive single tuned filter}

The passive single tuned filter (STF) is series RLC circuit that is most used in industry. Simple layout of passive STF is illustrated in Fig. 2. STF circuit is tuned to a resonance frequency. The concept of STF is tuned to suppress a simple frequency by given voltage level and fundamental frequency $(50 \mathrm{~Hz} / 60 \mathrm{~Hz})$. Thus, it reduces harmonics in current flowing to the load. The advantages of this type filters are simple, cheap and efficient at the tuned frequency. The disadvantage of STF is this filter cannot impact to high order harmonics.

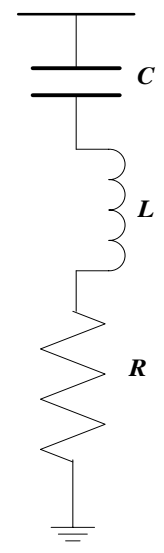

Fig. 2. Layout of passive single tuned filter

STF equivalent impedance equals as follows [13]

$$
Z_{f}=R_{f}+j\left(X_{L}-X_{c}\right)
$$

At resonance frequency

$$
X_{L}=X_{c}=2 \pi f_{r} L=\frac{1}{2 \pi f_{r} C}
$$

$X_{L}$ is the filter inductive reactance of inductance $L$.

$X_{C}$ is the filter capacitive reactance of capacitance $C$.

$X_{r}$ is the reactance at resonance frequency $f_{r}$.

And from equation (1)

$$
Z_{f}=R_{f}
$$

For the series connected STF the resonance frequency is chosen to be as $50 \mathrm{~Hz}$ and selecting a value of capacitance $C$ to be $50 \mu \mathrm{F}$ and from equation (1), $L$ is calculated to be $0.21 \mathrm{H}$. The parameters of designed SFT is given in Table 1 .

Table 1. Parameters of designed STF

\begin{tabular}{|c|c|c|}
\hline $\boldsymbol{C}(\boldsymbol{\mu F})$ & $\boldsymbol{L}(\boldsymbol{H})$ & $\boldsymbol{R}(\boldsymbol{O m})$ \\
\hline 50 & 0.21 & 7 \\
\hline
\end{tabular}




\section{Results and Discussions}

The standalone PV-based MG simulation in MATLAB/Simulink is shown in Fig. 3. The simulation is implemented in two cases: i) simulation without STF and ii) simulation with STF. Firstly, let's consider simulation results without STF.

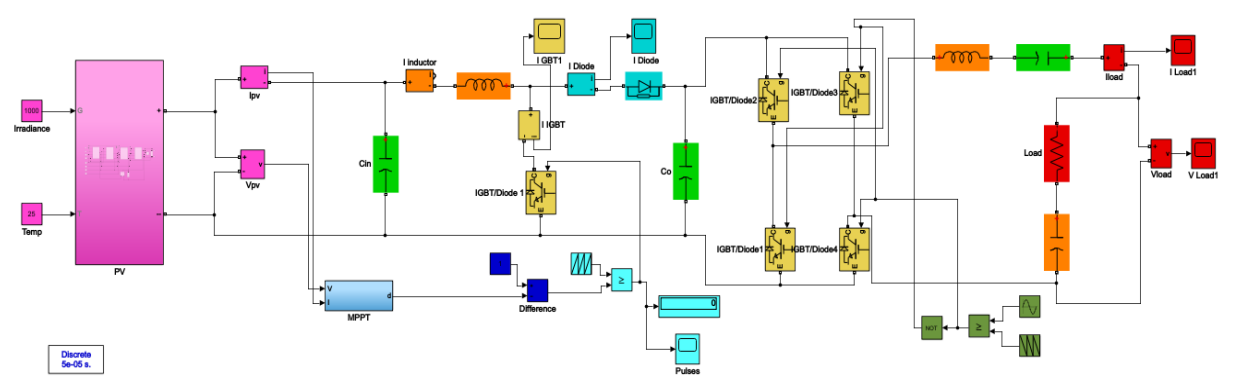

Fig.3. The simulation of PV-based MG

\subsection{Simulation Results without passive STF}

The output voltage, current and power of PV are shown in Fig.4, Fig.5, Fig.6, respectively.

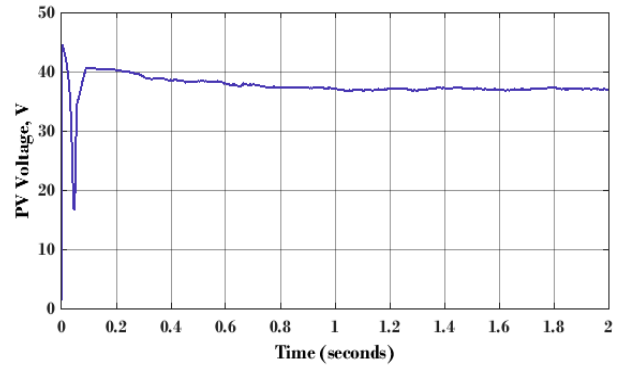

Fig. 4. PV output voltage

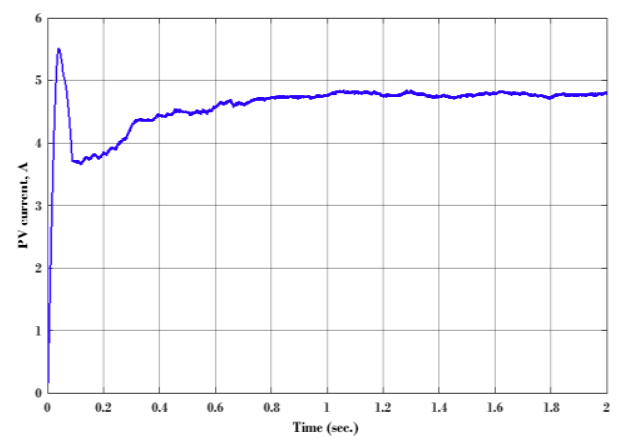

Fig. 5. PV output current

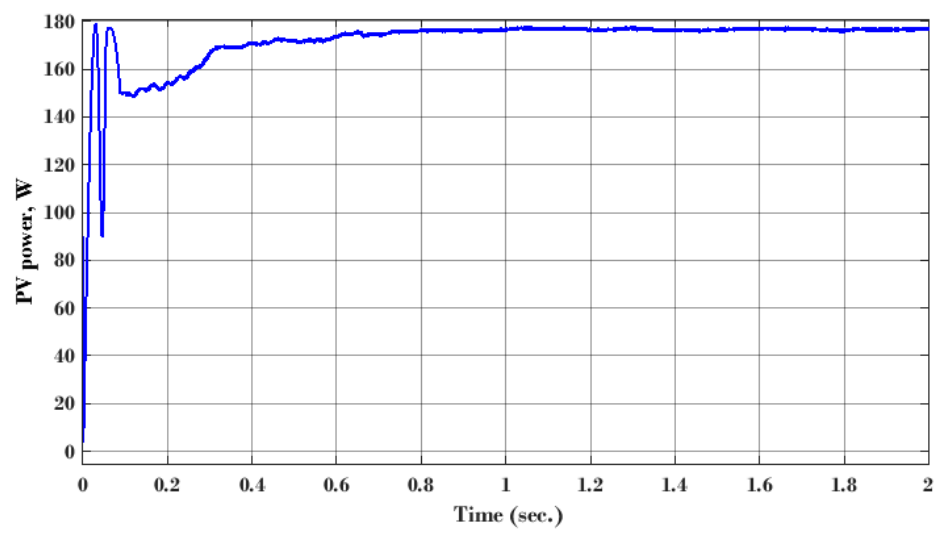

Fig. 6. PV Power 
The resistive load current and voltage without passive STF at power factor PF $=1$ are shown in Fig.7 and Fig.8, respectively. The frequency spectrums current and voltage are shown in Fig. 9 (a) and Fig.9 (b), respectively.

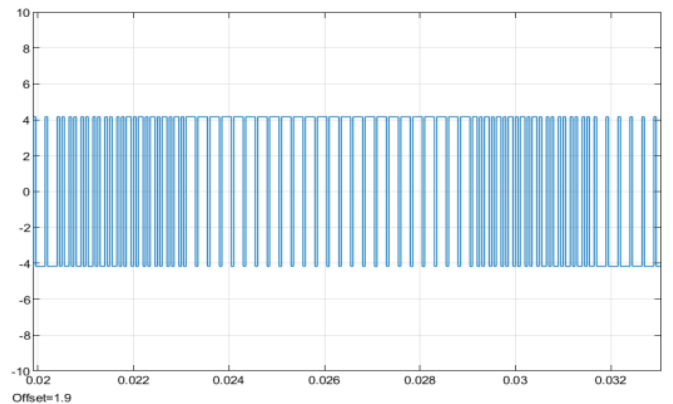

Fig. 7. Load current without STF

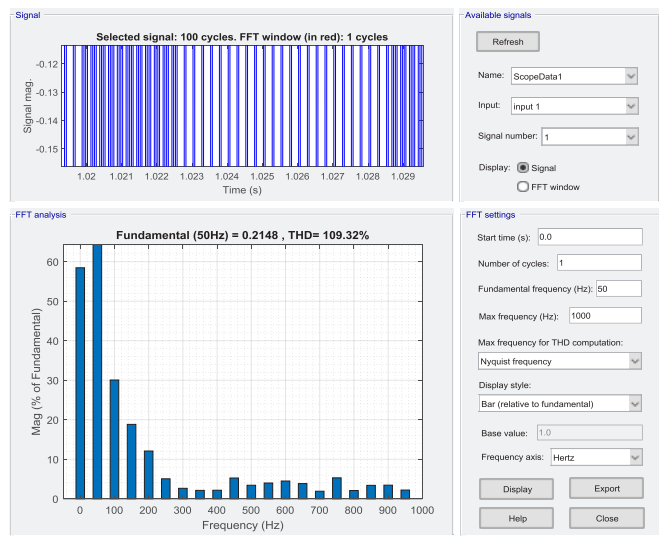

(a)

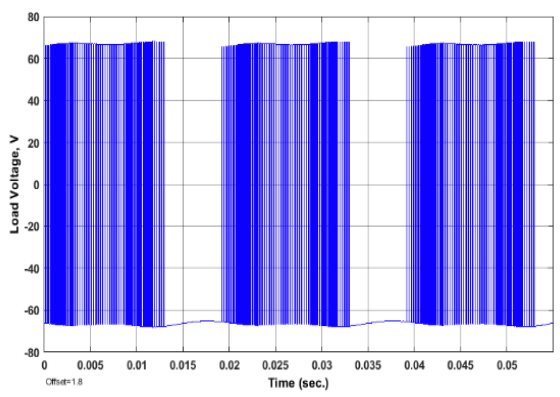

Fig. 8. Load voltage without STF
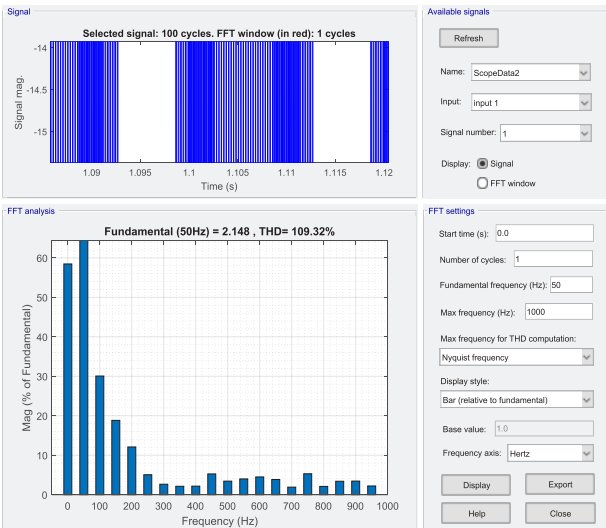

(b)

Fig. 9. (a) Current frequency spectrum without STF (b) Voltage frequency spectrum without STF

\subsection{Simulation Results with passive STF}

The inverter output current and voltage at load $\mathrm{PF}=1$ are shown in Figures 10 and 11, respectively. The frequency spectrum of the current and voltage at load $\mathrm{PF}=1$ are shown Fig. 12 (a) and Fig.12 (b), respectively.

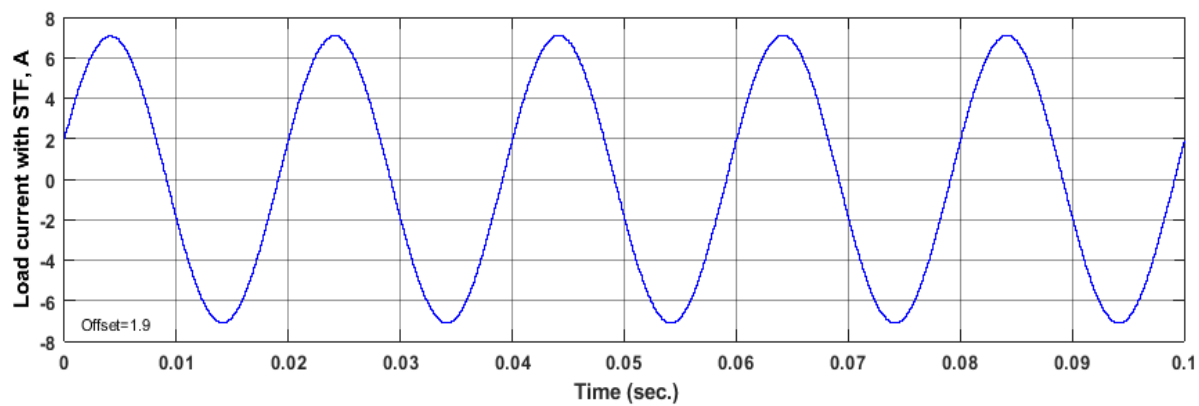

Fig. 10. Load current with STF 


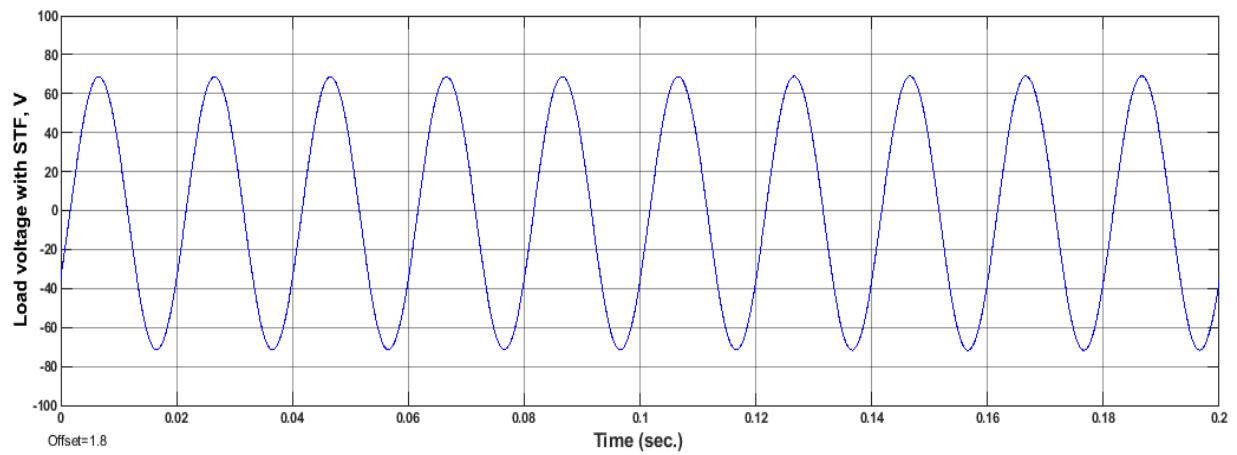

Fig. 11. Load voltage with STF

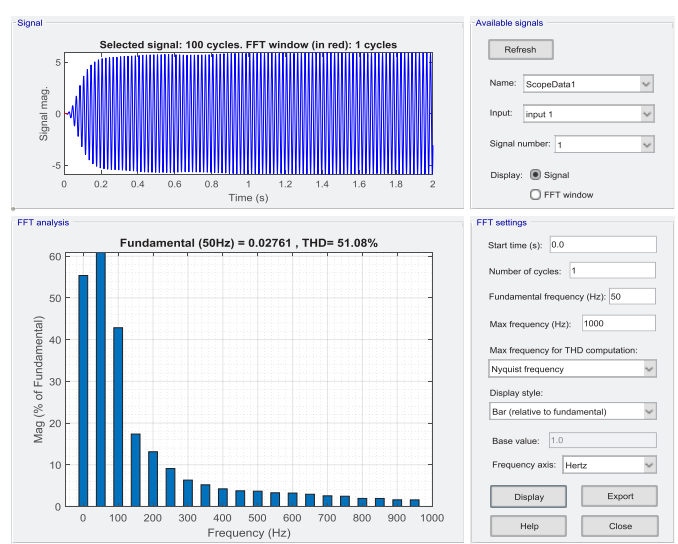

(a)
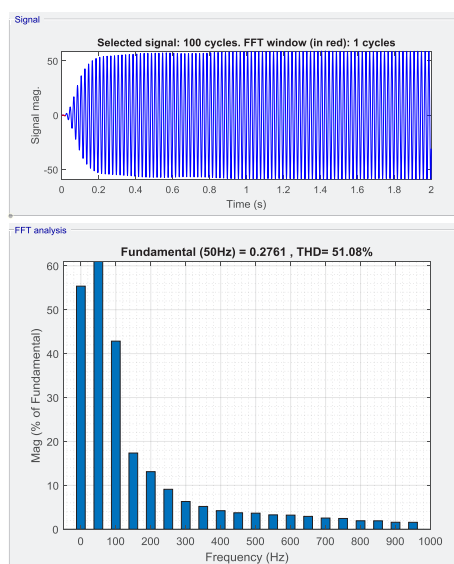

(b)

Fig. 12. (a) Current frequency spectrum at load $P F=1$; (b) Voltage frequency spectrum at load $P F=1$

The inverter output current and voltage at load of $\mathrm{PF}=0.9$ lag are shown in Fig. 13 and Fig. 14 , respectively. The frequency spectrum of the current and voltage at load of PF $=0.9$ lag are shown in Fig. 15 (a) and Fig.15 (b), respectively.

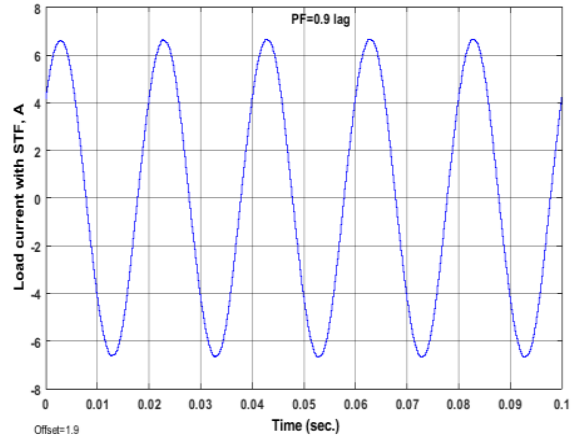

Fig. 13. Current at load $P F=0.9$ lag

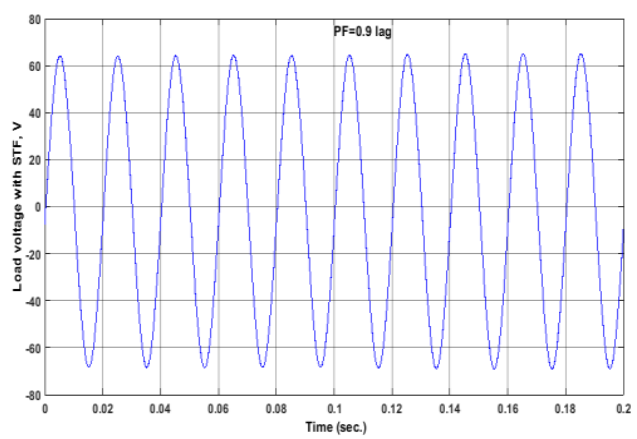

Fig. 14. Voltage at load $\mathrm{PF}=0.9$ lag 


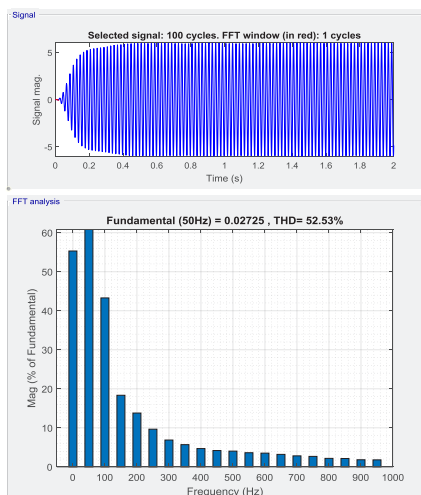

(a)
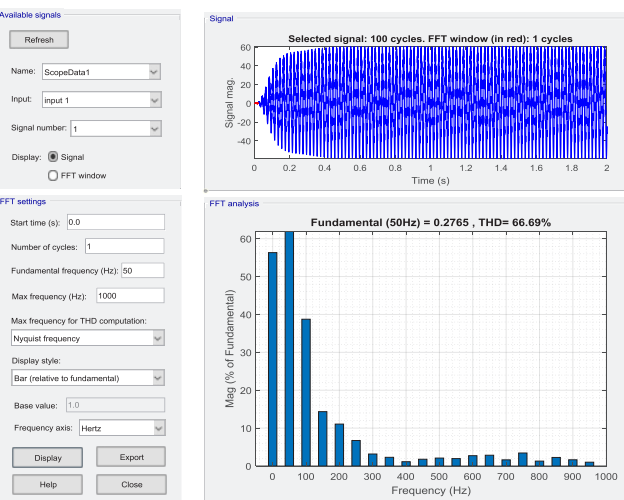

(b)

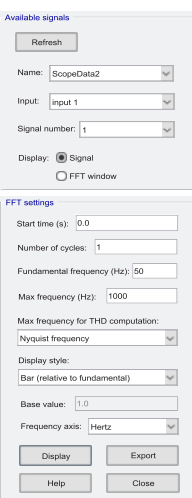

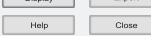

Fig. 15. (a) Current frequency spectrum at load PF $=0.9$ lag; (b) Voltage frequency spectrum at load $\mathrm{PF}=0.9$ lag

The inverter output current and voltage at load PF $=0.7$ lag are shown in Fig. 16 and Fig. 17, respectively. The frequency spectrum of the current and voltage at load $\mathrm{PF}=0.7$ lag are shown in Fig.18 (a) and Fig.18 (b), respectively.

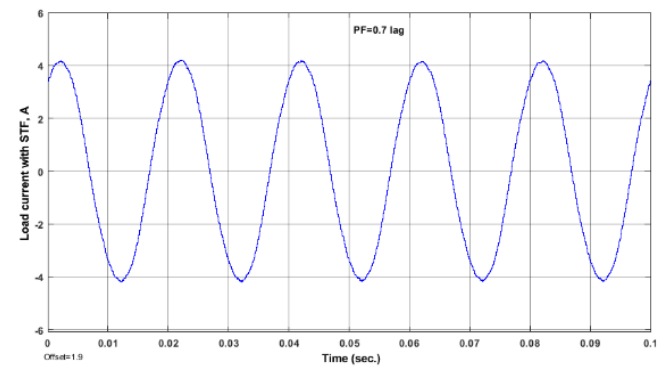

Fig. 16. Current at load $\mathrm{PF}=0.7 \mathrm{lag}$

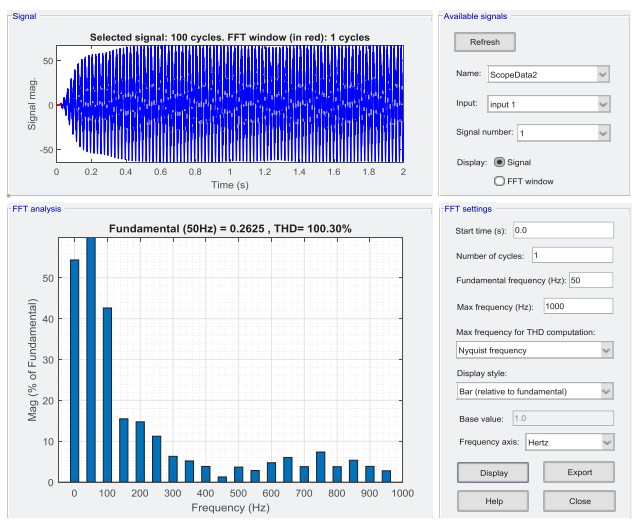

(a)

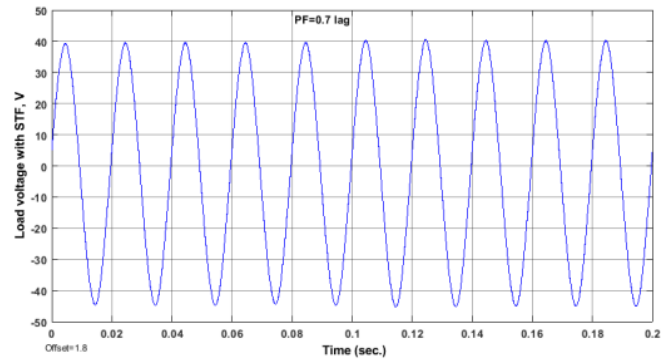

Fig. 17. Voltage at load $\mathrm{PF}=0.7 \mathrm{lag}$

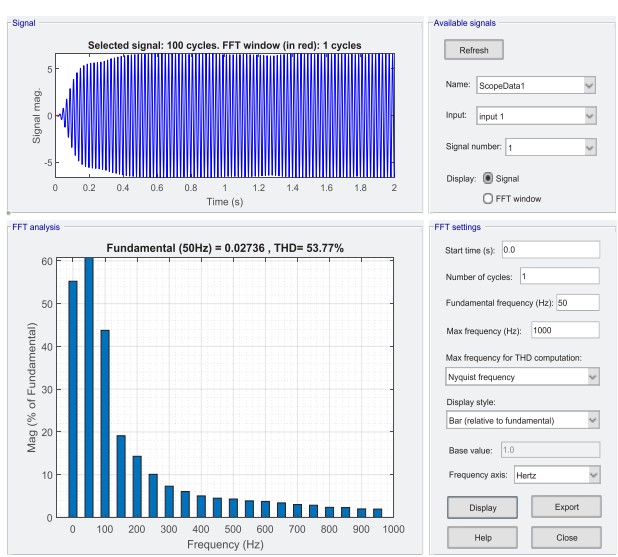

(b)

Fig. 18. (a) Current frequency spectrum at load $P F=0.7$ lag; (b) Voltage frequency spectrum at load $\mathrm{PF}=0.7 \mathrm{lag}$ 
The inverter output current and voltage at load $\mathrm{PF}=0.7$ lead are shown in Fig. 19 and Fig.20, respectively. The frequency spectrum of the current and voltage at load $\mathrm{PF}=0.7$ lead are shown in Fig. 21 (a) and 21 (b), respectively.

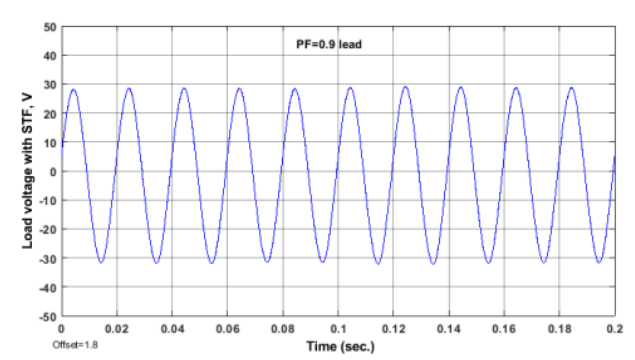

Fig. 19. Current at load $P F=0.7$ lead

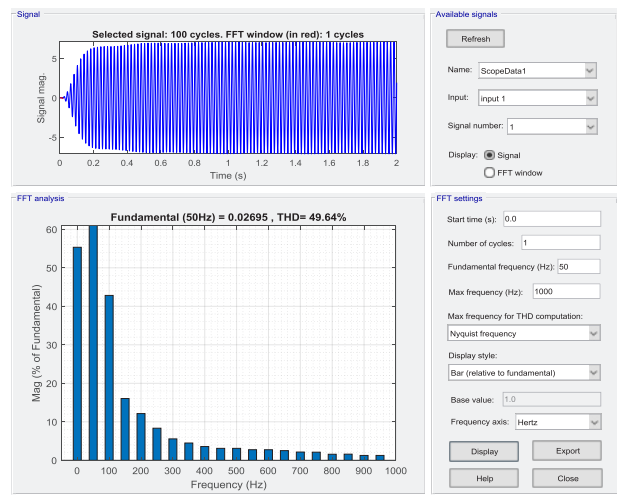

(a)

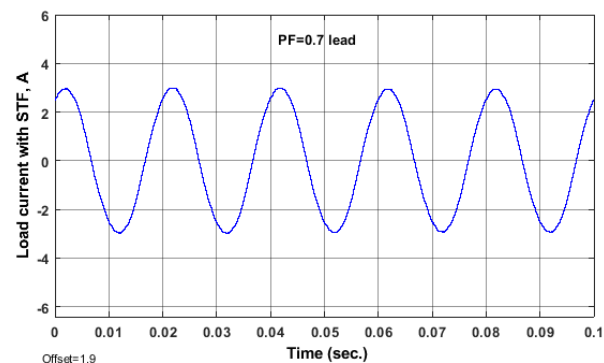

Fig. 20. Voltage at load $P F=0.7$ lead

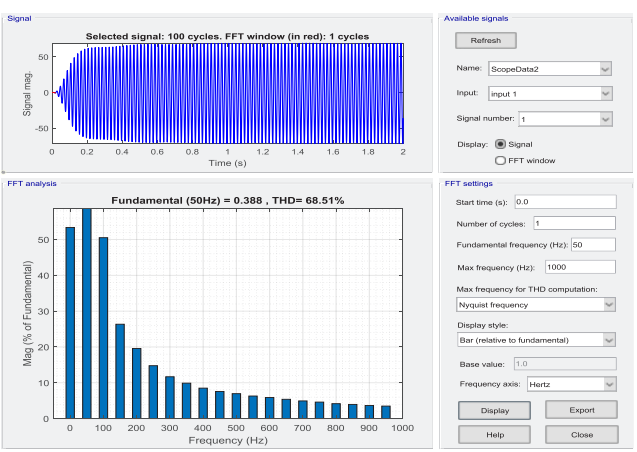

(b)

Fig. 21. (a) Current frequency spectrum at $P F=0.7$ lead; (b) Voltage frequency spectrum at $P F=0.7$ lead

Table 2 shows the values of current total harmonic distortion (ITHD) and voltage total harmonic distortion (VTHD) for the above simulated cases at different values of PF. It should ne notices, ITHD and VTHD values decrease by increasing lagging PF of load.

Table 2 Values of ITHD and VTHD with various load of PF

\begin{tabular}{|l|l|l|}
\hline PF & ITHD \% & VTHD \% \\
\hline 0.7 lag & 53.77 & 100.30 \\
\hline 0.9 lag & 52.53 & 66.69 \\
\hline 1 & 51.08 & 51.08 \\
\hline 0.7 lead & 49.64 & 68.51 \\
\hline
\end{tabular}

\section{Conclusions}

In PV-based MG system is utilized the passive STF to mitigate THD caused by non-linear loads. The comparison results of load current and voltage waveforms, and their frequency spectrums are illustrated that the power quality and energy efficiency of PV-based MG system are improved with passive STF. The simulation results show that the passive STF is effective in mitigating the harmonic distortion of the output voltage and current of PVbased MG with enhancing the output power quality and waveforms. The load PF is 
affecting the mitigation of the harmonic distortion and the effectiveness of the series connected STF as the STF resonance frequency is varying with the variation of the load PF.

\section{References}

1. H. Kanchev, F. Colas, V. Lazarov, B. Francois, IEEE TRANS. SUSTAIN Energy 5 (2014)

2. J. Klemeš, P. Varbanov, S. Pierucci, D. Huisingh, J Cleaner Prod 18 (2010)

3. Z. Yusupov, M. Guneser, INT J Energy APPL TECHN 3 (2016)

4. H. Jiayi, J. Chuanwen, X. Rong, Renew Sustain Energy Rev 12 (2008)

5. M. Mao, L. Cui, Q. Zhang, K. Guo, L. Zhou, H. Huang, Energy Reports 6, (2020)

6. P. Midya, P. Kerin, R. Turnbull, R. Reppa, J. Kimball, IEEE PESC Record 2 (1996)

7. A. Abdelsalam, M. Massoud, S. Ahmed, P. Enjeti, IEEE TRANS. POWER ELECTRON 26 (2011)

8. S. Hadji, J-P. Gaubert, F. Krim, Energy Procedia 74 (2015)

9. A. Sharma, Y. Koraz, M. Yousef, A novel control methodology for stand-alone photovoltaic systems utilizing maximum point power tracking, in Proceedings of the Canadian Conference on Electrical and Computer Engineering, CCECE, 5-8 May 2019, Toronto, Canada (2019)

10. A. Kusko, Power Quality in Power System (McGraw-Hil 2007)

11. J. Das, Power System Harmonics and Passive Filter Designs (IEEE 2015)

12. M. Tali, A. Obbadi, A. Elfajri, Y. Errami, J INT REN and SUST Energy 499-504 (2014)

13. A. Abbas, E. Ali, R. El-Sehiemy, A. El-Ela, K. Fetyan, Comprehensive Parametric Analysis of Single Tuned Filter in Distribution Systems, in Proceedings of International Middle East Power Systems Conference, MEPCON, 17-19 December 2019, Cairo, Egypt (2019) 